\title{
Topological defects caused by inhomogeneity of optical activity
}

\author{
Vasylkiv Yu., Skab I. and Vlokh R. \\ Vlokh Institute of Physical Optics, Dragomanov Street, 79005 Lviv, Ukraine, \\ vlokh@ifo.lviv.ua
}

Received: 12.04 .2017

\begin{abstract}
The present work is devoted to analysis of the effect of inhomogeneous axial fields (torsion and bending mechanical stresses, as well as conically shaped electric fields) on the optical activity in crystals belonging to different point symmetry groups. It is found that the topological defects of gyration surface orientation with half-integer strengths can appear under the action of these fields. We discuss potentials of spiral phase plates made of optically active materials for generating vortex vector beams.
\end{abstract}

Keywords: topological defects, optical activity, inhomogeneity, torsion, bending, electric field, spiral phase plates, vector beams, optical vortices

PACS: $78.20 . \mathrm{Ek}, 42.50 . \mathrm{Tx}, 78.20 . \mathrm{hb}, 78.20 . \mathrm{Jq}$

UDC: 535.56

\section{Introduction}

Topological defects (TDs) play important role in a number of natural phenomena, e. g. in the case of structural phase transitions [1], in the development of the early Universe [2,3] or in quantum communication systems [4]. The TDs arise in the material media of different types such as liquid crystals [5], quantum liquids (e.g., superfluid helium [6-9]), and ferroic materials [10]. For example, skyrmions and domain walls appearing in the course of phase transitions in ferroic materials play a role of TDs [1]. In liquid crystals and superfluid helium, TDs are also generated at a phase transition, since a modern theory of birth of the Universe involves this notion [2]. In other words, in all of the cases mentioned above the TDs result from some symmetry breaking that occurs under phase transitions.

The TDs in liquid crystals represent a specific spatial order-parameter field for which the order parameter cannot shrink to a point [11]. Perhaps, it is only the TDs in liquid crystals that can be induced by special processing of liquid crystalline cells and not by phase transitions. Recently, we have also shown that the TDs associated with the orientation of optical indicatrix in solid-state material media can be induced by various external factors, such as mechanical torsion [12, 13] or bending [14]. Moreover, the TDs can be produced by conically distributed electric fields, due to electrooptic Pockels and Kerr effect $[15,16]$. They can also arise due to piezooptic effect caused by the mechanical strains appearing around structural dislocations in solid crystals [17]. It would be natural to assume that the TDs of optical indicatrix orientation similar to those induced by the electrooptic Kerr effect can be generated using magnetic fields, due to a known Cotton-Mouton effect, since the structures of the relevant constitutive tensors are the same.

In particular, the TDs mentioned above can give rise to specific defects of the phase front of optical radiation transmitting through a medium and, therefore, result in generating optical vortices that bear a nonzero orbital angular momentum. It is interesting that optical activity effect decreases the efficiency of spin-to-orbit angular moment conversion [18]. Probably, this fact can be thought 
of as unique in the sense that here the optical activity plays an active part in producing the TDs and the corresponding optical vortices.

On the other hand, the optical activity as a phenomenon of spatial dispersion can cause quite different implications in the processes of appearance of the TDs. Namely, an inhomogeneous spatial distribution of the gyration tensor that describes the optical activity effect can itself contain the TDs. In the present we analyze the TDs associated with the gyration tensor and the optical rotation caused by the gyration, whenever the latter are induced by the torsion and bending stresses or by the conically distributed electric fields.

\section{Results and discussion}

\subsection{General remarks}

The optical activity is known to be associated with the spatial dispersion effect. It is described by a symmetric second-rank axial tensor $g_{k l}$ that arises as a result of accounting for dependence of the optical-frequency impermeability tensor $B_{i j}$ on the wave vector $k_{l}$ of optical wave:

$$
B_{i j}=B_{i j}^{0}+i e_{i j k} g_{k l} k_{l} .
$$

Here $B_{i j}^{0}$ denotes the optical-frequency impermeability with no spatial dispersion taken into account, while $e_{i j k}$ is the unitary, fully antisymmetric third-rank axial tensor termed as the LeviCivita tensor. Notice that the gyration tensor $g_{k l}$ is equal to zero in any centrosymmetric media.

Below we will consider the optical activity effects induced by mechanical stresses (a piezogyration) or electrical fields (an electrogyration). Therefore we must recall general relations that quantize the said effects [19]. The piezogyration effect is described as a change $\Delta g_{k l}$ in the gyration tensor appearing under the action of the mechanical stress $\sigma_{m n}$ :

$$
\Delta g_{k l}=\beta_{k l m n} \sigma_{m n},
$$

where $\beta_{k l m n}$ is the fourth-rank axial tensor with the internal symmetry $\varepsilon\left[\mathrm{V}^{2}\right]^{2}$. This symmetry implies that the piezogyration can exist only in non-centrosymmetric media. The linear electrogyration is described as

$$
\Delta g_{k l}=\gamma_{k l m} E_{m}
$$

where $\gamma_{k l m}$ is the third-rank axial tensor with the internal symmetry $\varepsilon\left[\mathrm{V}^{2}\right] \mathrm{V}$ and $E_{m}$ the strength of the electric field. The effect can exist in crystalline materials belonging to almost all the symmetry groups.

\subsection{Topological defects of gyration tensor orientation}

Since we consider the optical activity induced by the mechanical torsions, the bending stresses and the conically distributed electric fields, there is a need in clarifying analytical relations describing the spatial distributions of components of the mechanical stress tensor and the electric field vector. It is known [20] that, under torsion of a cylindrical crystalline sample around its geometrical axis, there appears a shear stress which may be presented as

$$
\sigma_{\mu}=\frac{2 M_{Z}}{\pi R^{4}}\left(X \delta_{4 \mu}-Y \delta_{5 \mu}\right) \text {. }
$$

In Eq. (4), the axis $Z$ coincides with the torsion axis, the axes $X$ and $Y$ are orthogonal to $Z$, $M_{Z}=\int_{S}(r \times P) d S$ denotes the torsion torque, $\delta_{v \mu}$ the Kronecker delta, $R$ the cylinder radius, $S$ the 
square of the cylinder basis, and $P$ the mechanical load. As a result, we have two nonzero shear components of the stress tensor, $\sigma_{4}$ and $\sigma_{5}$. Let the torsion moment be applied around either an optic axis of uniaxial crystals or a three-fold symmetry axis of crystals that belong to the cubic system. As an example, the piezogyration tensor for the symmetry group $\overline{6} \mathrm{~m} 2$ acquires the following form [19]:

\begin{tabular}{c|cccccc} 
& $\sigma_{11}$ & $\sigma_{22}$ & $\sigma_{33}$ & $\sigma_{32}$ & $\sigma_{31}$ & $\sigma_{21}$ \\
\hline$g_{11}$ & 0 & 0 & 0 & 0 & $\beta_{1131}$ & 0 \\
$g_{22}$ & 0 & 0 & 0 & 0 & $-\beta_{1131}$ & 0 \\
$g_{33}$ & 0 & 0 & 0 & 0 & 0 & 0 \\
$g_{32}$ & 0 & 0 & 0 & 0 & 0 & $\beta_{3221}$ \\
$g_{31}$ & $\beta_{3221}$ & $-\beta_{3221}$ & 0 & 0 & 0 & 0 \\
$g_{21}$ & 0 & 0 & 0 & $-\beta_{1131}$ & 0 & 0
\end{tabular}.

Note that the crystals belonging to this symmetry group manifest no natural optical activity. Thus, the gyration tensor components induced by the torsion stresses can be written as $g_{11}=\beta_{1131} \sigma_{31}, g_{22}=-\beta_{1131} \sigma_{31}$ and $g_{21}=-\beta_{1131} \sigma_{32}$. As seen from Fig. 1a, the indicative surface of the gyration tensor (or simply a 'gyration surface') has an oval-like shape, with its petals revealing different signs of the effect. The angle $\zeta_{Z}$ of rotation of the gyration surface around the $Z$ axis (i.e., the optic axis) is given by

$$
\tan 2 \zeta_{Z}=\frac{2 g_{21}}{g_{11}-g_{22}}=-\frac{\sigma_{32}}{\sigma_{31}} .
$$

Taking into account Eq. (4) and the evident relations $X=\rho \cos \varphi$ and $Y=\rho \sin \varphi$ for the polar coordinate system (with the angle $\varphi$ being counted out anticlockwise, beginning from the positive $X$ axis), one can rewrite Eq. (6) as

$$
\tan 2 \zeta_{Z}=\frac{X}{Y}=\tan \left(\frac{\pi}{2}-\varphi\right), \quad \zeta_{Z}=\frac{\pi}{4}-\frac{\varphi}{2} .
$$

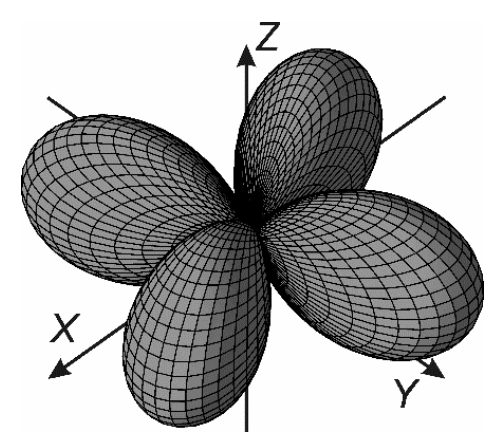

(a)

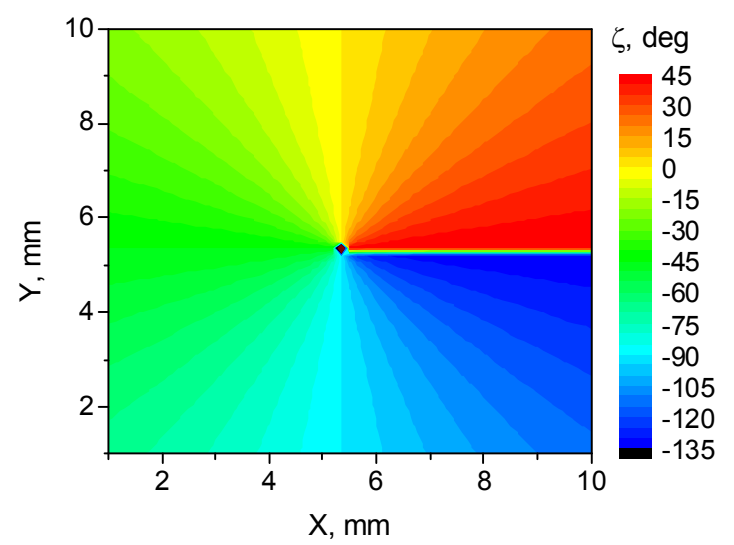

(b)

Fig. 1. The shape of gyration surface induced by the torsion stress (a), and (b) - XY distribution of rotation angle of the gyration eigenvectors around the $Z$ axis. 
Let us analyze the rotation of the gyration surface under the conditions when a wide parallel optical beam propagates through the sample along the $Z$ axis. Then the dependence of the angle $\zeta_{Z}$ upon the tracing angle $\varphi$ is linear and the eigenvectors of the gyration tensor rotate by $180 \mathrm{deg}$ around the $Z$ axis whenever the tracing angle change amounts to $360 \mathrm{deg}$. Then the $X Y$ distribution of the gyration tensor arising under the torsion reveals a TD with the strength equal to $1 / 2$ (see Fig. 1b). Note that a similar TD of the gyration tensor, with the same half-integer strength, should be observed for the crystals that belong to the symmetry groups $\overline{4} 3 \mathrm{~m}, 3 \mathrm{~m}, 3,32$ and $\overline{6}$.

Now we proceed to analyze the TDs of the gyration surface appearing when the bending stress is applied and the load $P_{1}$ is distributed over the upper surface of a crystal sample. When the sample is loaded along the $X$ axis, the two stress tensor components come to play (see Ref. [14]):

$$
\sigma_{22}=\frac{M}{J_{Z}} X=\frac{6 q}{b h^{3}}\left(\frac{h^{2}}{4}-Y^{2}\right) X, \quad \sigma_{21}=\frac{Q}{2 J_{Z}}\left(\frac{h^{2}}{4}-X^{2}\right)=-\frac{6 q}{b h^{3}}\left(\frac{h^{2}}{4}-X^{2}\right) Y .
$$

Here $J_{Z}$ is the area moment of inertia, $M$ the bending moment, $b, h$ and $l$ imply respectively the width, the height and the length of the crystalline beam, and the $q$ parameter is defined as $q=P_{1} / l$. It is easy to prove that the TD of the gyration surface orientation can appear due to bending in all of the non-centrosymmetric symmetry groups that contain only rotation symmetry axes among their symmetry operations, as well as in the groups $6 \mathrm{~mm}, 4 \mathrm{~mm}$ and $3 \mathrm{~m}$. For example, the angle of rotation of the gyration surface around the $Z$ axis for the group $6 \mathrm{~mm}$ reads as

$$
\tan 2 \zeta_{Z}=\frac{2 g_{21}}{g_{11}-g_{22}}=\frac{\sigma_{22}}{\sigma_{21}} .
$$

If we have $X, Y \rightarrow 0$ and certain conditions are satisfied for the size of the crystalline beam (see Ref. [14]), the relations $\tan 2 \zeta_{Z}=\cot \varphi$ and $\zeta_{Z}=\frac{\pi}{4}-\frac{\varphi}{2}$ hold true. Thus, in all of the cases mentioned above the TDs have half-integer strengths.

As shown in the work [15], the conically shaped electric field results in the following coordinate dependences of the electric field components:

$$
E_{1}=\frac{U}{d} \frac{\tan \Theta}{1+\tan ^{2} \Theta} \cos \varphi, \quad E_{2}=\frac{U}{d} \frac{\tan \Theta}{1+\tan ^{2} \Theta} \sin \varphi, \quad E_{3}=\frac{U}{d\left(1+\tan ^{2} \Theta\right)},
$$

where $U$ is the electric voltage applied, $d$ the thickness of an electrooptic cell, and $X=\rho \sin \Theta \cos \varphi, \quad Y=\rho \sin \Theta \sin \varphi$ and $Z=\rho \cos \Theta$ correspond to the spherical coordinate system. Issuing from the structure of the electrogyration tensor for different symmetry groups, it follows that the TD of orientation of the gyration surface arising under the conical electric field can appear only in those crystals that belong to the trigonal system, i.e. to the symmetry groups 32 , $3,3 \mathrm{~m}, \overline{3} \mathrm{~m}$ and $\overline{3}$. As an example, the rotation angle of the gyration surface for the group $3 \mathrm{~m}$ is given by

$$
\tan 2 \zeta_{Z}=\frac{2 g_{21}}{g_{11}-g_{22}}=-\frac{E_{2}}{E_{1}}=\tan (\pi-\varphi), \quad \zeta_{Z}=\pi / 2-\varphi / 2 .
$$

As a consequence, the conically shaped electric field produces the TD of the gyration surface orientation with the half-integer strength. This remains true for all the symmetry groups mentioned above. 
Hence, we have demonstrated that, under the action of inhomogeneous external fields, the $X Y$ distribution of the gyration tensor manifests singular points which give birth to the linear TDs of the gyration tensor orientation. However, contrary to the earlier known TDs associated with the orientation of optical indicatrix, the TDs of the gyration surface orientation do not affect the parameters of light propagating along the $Z$ axis. In fact, the latter parameters are influenced only by the cross-section $X Y$ of the optical indicatrix. Strict consideration of the influence of optical activity on the parameters of light propagating along the $Z$ axis testifies that the TDs are affected only by the gyration component $g_{33}$.

Unfortunately, the heterogeneous external fields dealt with here either cannot change the $g_{33}$ component for the crystals of middle and cubic systems or, alternatively, can change this component but with no appearance of the TDs. In such a case the following question arises: which kind of material inhomogeneity can lead to the appearance of TD of the gyration tensor, which generates defects in the phase front of the light emergent from crystal? At this point we remind that the optical gyration governs the phase difference $\alpha$ between the left- and right-handed circularly polarized waves:

$$
\alpha=2 \chi=2 \rho d_{\mathrm{Z}}=\frac{2 \pi d_{\mathrm{Z}} g_{33}}{\lambda n_{\mathrm{Z}}},
$$

where $\chi$ is the angle of polarization plane rotation, $\rho$ the specific rotation angle of the polarization plane, $d_{\mathrm{z}}$ the sample thickness along the $Z$ direction, $\lambda$ the light wavelength, and $n_{\mathrm{Z}}$ the refractive index.

The suggestion mentioned above implies that, in order to generate an optical vortex exclusively via the TD of optical activity, the phase profile caused by the gyration has to be helical. The easiest method for achieving such a helical phase front is to utilize a spiral phase plate prepared from optically active material and a circularly polarized incident light wave. However, simple estimations demonstrate that the phase difference $2 \pi$ due to the optical activity needed for generating, e. g., at a helix pitch $\sim 1 \mu \mathrm{m}$ is equal to $g_{33} \approx 1$ (with $n_{\mathrm{z}}=1.5$ and $\lambda=632.8 \mathrm{~nm}$ ). It is too high optical activity, which is never observed in single crystals. As far as we know, the largest $g_{33}$ values occurring in the crystalline materials such as $\left(\mathrm{Ga}_{0.6} \mathrm{In}_{0.4}\right)_{2} \mathrm{Se}_{3}$ and $\mathrm{LiIO}_{3}$ are of the order of $\sim 10^{-3}$ only $[19,21]$. Moreover, the large enough optical activity in need cannot be induced by any known external field, e. g., due to electrogyration or piezogyration. Anyway, one can assume that it can be realized with some artificial materials. Then initially linearly polarized incident optical beam can acquire a vector-vortex beam, e.g., because of a helical shape of the outgoing face of optically active sample. Then the optical vortex is generated mainly due to the appearance of a 'classical' phase difference for the light passing through the spiral crystal plate, while the vector structure of this beam appears due to the presence of optical activity. In this case a doughnut mode-like spatial distribution of polarization states in the emergent vortex beam would be azimuthal, as shown in Fig. 2. This implies that, in principle, the optical gyration effect can

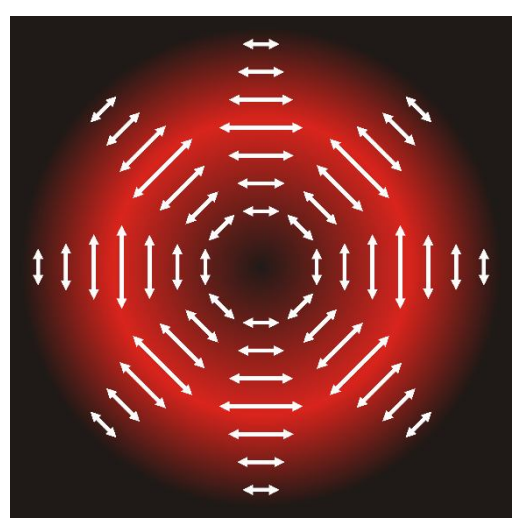

Fig. 2. Schematic azimuthal distribution of polarization states in the emergent vortex beam bearing a doughnut mode. 
be used for generating vector beams. However, the latter assertion needs a much more detailed analysis. In particular, it is not clear what would happen if, under the condition of full-circle angular tracing, the angle of polarization plane rotation is not a multiple of $\pi$. These problems will be a subject of our forthcoming studies.

\section{Conclusion}

In the present work we have analyzed the effect of inhomogeneous mechanical stresses and electric fields on the optical activity of crystals belonging to different point groups of symmetry. We have found that, like the application of mechanical torsion and bending or conically shaped electric fields to solid crystals can produce the TDs of gyration tensor orientation. In all of the cases under study, the strength of the TDs is half-integer. We have also shown that these topological defects do not affect the parameters of light that propagates along the dislocation line. Besides, the torsion and bending stresses with their moments parallel to the high-fold symmetry axis, as well as the conically shaped electric fields with the cone axes parallel to the said direction, cannot lead to appearance of singular spatial distributions of the gyration parameters, which are responsible for the light propagating along the dislocation line. At the same time, we have demonstrated that the spiral phase plate made of 'artificial' optically active materials, in principle, can be used for forming the vortex-vector optical beams.

\section{References}

1. Seidel J. Topological structures in ferroic materials. Domain walls, vortices and skyrmions. Springer International Publishing (2016).

2. Zurek W H, 1985. Cosmological experiments in superfluid helium? Nature. 317: 505-508.

3. Trebin H-R, 1998. Defects in liquid crystals and cosmology. Liquid Cryst. 24: 127-130.

4. Nagali E, Sciarrino F, De Martini F, Marrucci L, Piccirillo B, Karimi E and Santamato E, 2009. Quantum information transfer from spin to orbital angular momentum of photons. Phys. Rev. Lett. 103: 013601.

5. Kleman M and Lavrentovich O D, 2006. Topological point defects in nematic liquid crystals. Phil. Mag. 86: 4117-4137.

6. Salomaa M M and Volovik G E, 1987. Quantized vortices in superfluid ${ }^{3} \mathrm{He}$. Rev. Mod. Phys. 59: 533-613.

7. Kurik M V and Lavrentovich O D, 1988. Defects in liquid crystals: homotopy theory and experimental studies. Sov. Phys.: Uspekhi. 31: 196-224.

8. Onsager L, 1949. The two fluid model for helium II [remarks by L. Onsager on pp. 249-250]. Nuovo Cimento, Suppl. 6: 249-250.

9. Feynman R. In: Progress in Low Temperature Physics, Vol. 1. Ed. by C Gorter. NorthHolland (1955).

10. Lin S-Z, Wang X, Kamiya Y, Chern G-W, Fan F, Fan D, Casas B, Liu Y, Kiryukhin V, Zurek W H., Batista C D and Cheong S-W, 2014. Topological defects as relics of emergent continuous symmetry and Higgs condensation of disorder in ferroelectrics. Nature Phys. 10: 970-977.

11. Mermin N D, 1979. The topological theory of defects in ordered media. Rev. Mod. Phys. 51: 591-648.

12. Skab I, Vasylkiv Yu, Savaryn V and Vlokh R, 2011. Optical anisotropy induced by torsion stresses in $\mathrm{LiNbO}_{3}$ crystals: appearance of an optical vortex. J. Opt. Soc. Amer. A. 28: 633-640. 
13. Skab I, Vasylkiv Yu, Zapeka B, Savaryn V and Vlokh R, 2011. Appearance of singularities of optical fields under torsion of crystals containing threefold symmetry axes. J. Opt. Soc. Amer. A. 28: $1331-1340$.

14. Skab I, Vasylkiv Yu and Vlokh R, 2012. Induction of optical vortex in the crystals subjected to bending stresses. Appl. Opt. 51: 5797-5805.

15. Skab I, Vasylkiv Yu, Smaga I and Vlokh R, 2011. Spin-to-orbital momentum conversion via electro-optic Pockels effect in crystals. Phys. Rev. A. 84: 043815.

16. Vasylkiv Yu, Skab I and Vlokh R, 2014. Generation of double-charged optical vortices on the basis of electro-optic Kerr effect. Appl. Opt. 53: B60-B73.

17. Savaryn V, Vasylkiv Yu, Krupych O, Skab I and Vlokh R, 2013. Polarization singularities of optical fields caused by structural dislocations in crystals. J. Opt. 15: 044023.

18. Vasylkiv Yu, Krupych O, Skab I and Vlokh R, 2011. On the spin-to-orbit momentum conversion operated by electric field in optically active $\mathrm{Bi}_{12} \mathrm{GeO}_{20}$ crystals. Ukr. J. Phys. Opt. 12: $171-179$

19. Vlokh O G, Spatial dispersion phenomena in parametrical crystal optics, Lviv: Vyshcha Shkola (1984).

20. Sirotin Y and Shaskolskaya M P. Fundamentals of crystal physics. Moscow: Nauka (1975).

21. Kranjcec M, Desnica D I, Celustka B, Borec A N, Kovacs G Sh, Hadmashy Z P, Suslikov L M and Studenyak I P, 1996. On some crystal-optic properties of $\gamma-\left(\mathrm{Ga}_{\mathrm{x}} \mathrm{In}_{1-\mathrm{x}}\right)_{2} \mathrm{Se}_{3}$ single crystals. Phys. Stat. Solidi (a). 153: 539-546.

Vasylkiv Yu., Skab I. and Vlokh R. 2017. Topological defects caused by inhomogeneity of optical activity. Ukr.J.Phys.Opt. 18: 95 - 101

Анотація. У роботі аналізовано вплив неоднорідних аксіальних полів (крутильних $i$ згинаючих механічних напружень, а також конічного електричного поля) на оптичну активність кристалів, що належать до різних точкових груп симетрії. Виявлено, щео під дією иих полів виникатимуть топологічні дефекти орієнтації гіраційної поверхні $з$ напівиілим зарядом. Обговорено можливість використання спіральних фазових пластинок, виготовлених з оптично активних матеріалів, для формування вихрових векторних пучків. 